\title{
Identification and Clinical Characterization of Children with Benign Ethnic Neutropenia
}

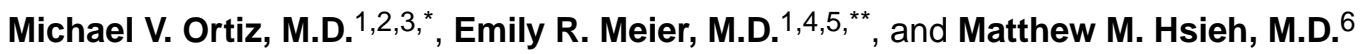 \\ ${ }^{1}$ Center for Cancer and Blood Disorders, Children's National Medical Center, Washington, DC \\ 2 Department of Pediatrics, Memorial Sloan Kettering Cancer Center, New York City, NY \\ ${ }^{3}$ Division of Pediatric Hematology and Oncology, New York Presbyterian / Weill Cornell Medical \\ Center, New York City, NY \\ ${ }^{4}$ Department of Pediatrics, The George Washington School of Medicine and Health Sciences, \\ Washington, DC \\ 5 Indiana Hemophilia and Thrombosis Center, Indianapolis, IN \\ ${ }^{6}$ Molecular and Clinical Hematology Branch, National Institute of Diabetes, Digestive, and Kidney \\ Diseases (NIDDK) and National Heart, Lung, and Blood Institute (NHLBI), National Institutes of \\ Health (NIH), Bethesda, MD
}

\begin{abstract}
Benign ethnic neutropenia (BEN) is an asymptomatic condition reported in adults of African and Middle Eastern descent. The clinical description in children is currently lacking. In our urban outpatient pediatric hematology clinic, the median neutrophil count of children with BEN was lower than previous reports in adults at $893 \times 10^{6} \mathrm{cells} / \mathrm{L}$, but increased with older age. There was an equal male to female ratio and $24 \%$ of our BEN children reported ethnicities other than African or Middle Eastern. Children with BEN had a clinical course comparable to other healthy children including otherwise normal blood counts, except for mild anemia.
\end{abstract}

\section{Keywords}

Neutropenia; Benign Ethnic Neutropenia; Pediatric Hematology/Oncology

\section{Introduction}

Benign ethnic neutropenia (BEN), also known as benign familial neutropenia, benign neutropenia of the black, and chronic benign idiopathic neutropenia, is an asymptomatic

\footnotetext{
Corresponding Author: Michael V. Ortiz, M.D., Memorial Sloan Kettering Cancer Center, 1275 York Ave, Box 139, New York, NY 10065, Phone: 212-639-7575, Fax: 212-717-3107, ortizm2@mskcc.org.

* Current affiliations: Memorial Sloan Kettering Cancer Center and New York Presbyterian / Weill Cornell Medical Center, New York, NY

* Current affiliation: Indiana Hemophilia and Thrombosis Center, Indianapolis, IN

Conflicts of Interest: The authors have no conflicts of interest and have all made significant contributions to the manuscript. This project is approved by all authors, has not been published previously, and is not under consideration for publication elsewhere.
} 
condition commonly observed in individuals of African, Arab, as well as Ethiopian and Yemenite Jewish descent. ${ }^{1-6}$ Admixture mapping of individuals of African and Yemenite Jewish ancestry implicates a single nucleotide polymorphism (SNP) in the promoter region of the Duffy antigen and receptor for chemokine (DARC) as a heritable trait associated with BEN. ${ }^{7-9}$ This SNP has been reported to modulate infectivity from Plasmodium species, and more recently with HIV. ${ }^{3,7,8}$ The effect of the DARC SNP on Plasmodium infectivity and geographical distribution of BEN, which overlaps that of thalassemia and sickle cell disease, have led researchers to suggest that widespread prevalence may be the result of a historical balanced polymorphism. ${ }^{2}$ Although the precise mechanism of the survival benefit of neutropenia in this setting is unknown, Denic and colleagues hypothesized that the impairment in the ability of BEN individuals to release the neutrophils into the peripheral blood may have conferred a survival advantage due to decreased tissue damage from chronic malarial infections. ${ }^{2}$

BEN is defined in adults by recurrent absolute neutrophil count (ANC) less than $1,500 \times 10^{6}$ cells/L in the absence of other secondary causes of neutropenia, such as infections, drugs, cancer, autoimmune diseases, metabolic disturbances, and hematologic disorders.

Individuals with BEN have normal myeloid maturation within the bone marrow but release fewer neutrophils into the peripheral circulation in response to steroid or epinephrine stimulation. ${ }^{4-6} \mathrm{BEN}$ is a clinically distinct entity from other congenital neutropenias such as severe congenital neutropenia, cyclic neutropenia, and chronic idiopathic neutropenia. These latter diseases are characterized by frequent severe neutropenia (ANC less than $500 \times 10^{6}$ cells/L) and oral mucosal or systemic infections. Previous studies of BEN in pediatrics reported no increased infections but otherwise lacked detailed demographic, clinical, and laboratory descriptions. ${ }^{1,10-12}$ The purpose of this study is to address the need to further characterize the clinical features of this condition in children.

\section{Materials and Methods}

A single institution retrospective study from 2008-2013 was conducted at an urban tertiary care children's hospital in Washington, DC, after the Institutional Review Board granted a waiver of consent. Potential BEN individuals were initially identified by searching for ICD9 codes 288.0 (neutropenia) and 288.09 (neutropenia, not otherwise specified). ANC less than $1,500 \times 10^{6}$ cells/L was required for inclusion. Exclusion criteria included pregnancy, low B12 or folate levels, other hematologic disorders, cancer, or concurrent treatment with immunosuppressants. Additionally, patients with neutropenia concurrent with a viral infection that self-resolved or patients that had neutropenia at their primary care provider's office but had a normal ANC when evaluated in the pediatric hematology clinic were also excluded. All outpatient visits to the pediatric hematology clinic in which a documented ANC was obtained were evaluated and recorded as encounters for patients included in the BEN cohort. Individuals were included in the BEN cohort even if they had one or more visits to the pediatric hematology clinic, regardless of length of follow-up. All cases were reviewed by two members of the study team to confirm the validity of the designation of BEN. Demographic, laboratory, and clinical information on all included patients were recorded from each clinic encounter. Potential clinical sequelae of prolonged neutropenia that were collected included upper respiratory infections, rash, poor weight gain, weight 
loss, headaches, oral ulcers, bone pains, joint pains, cervical lymphadenopathy, gingivitis, dental problems, night sweats, chills, delayed wound healing, and dyspnea. Laboratory information collected included complete blood counts, immunoglobulin levels, bone marrow aspirate or biopsy results, as well as evaluations for infections, autoimmune disorders, or inherited bone marrow failure syndromes.

The medians and $25^{\text {th }}$ and $75^{\text {th }}$ percentiles were calculated for each laboratory parameters (Table 3) of all BEN patients. BEN patients were then stratified into 3 age groups, 0-6 years $(\mathrm{N}=26), 6-12$ years $(\mathrm{N}=6)$, and 12-18 years $(\mathrm{N}=5)$. WBC and ANC were compared between age groups, and between African descent vs all other ethnicities using Wilcoxon/KruskalWallis test, with normal or Chi Square approximation, and $\mathrm{p}<0.05$ considered to be statistically significant.

\section{Results}

\section{Demographic Characteristics}

Searching by ICD9 codes yielded 136 neutropenic individuals. Ninety-nine were excluded: 43 had anti-neutrophil antibodies consistent with autoimmune neutropenia, 26 had transient or self-resolved neutropenia, 22 had a concurrent malignancy, 4 were receiving immunosuppressant therapy, and 4 had other known causes of neutropenia. Among the 26 children with transient neutropenia, 6 had viral infections concurrent with neutropenic blood counts. The remaining 20 individuals had a complete blood count (CBC) at the referring pediatrician's office which identified neutropenia, and a repeat $\mathrm{CBC}$ at our clinic with normal neutrophil count. None of these patients were referred back to us or had additional CBCs available to us at our clinic or elsewhere to determine if subsequent CBCs revealed neutropenia again.

The remaining 37 individuals comprised our BEN cohort (Table 1). A total of 130 encounters in the outpatient pediatric hematology clinic occurred for these 37 children, accounting for 70.4 patient-years of follow-up. Median age at evaluation was 1.8 years (range 0.1-17.3); median duration of follow-up was 16.1 months (range 0.8 to 2.8 months). Ten patients had 1 encounter or less than 6 months of follow-up. This group with a limited duration of follow-up were all determined to have BEN by the treating pediatric hematologist and confirmed by another senior pediatric hematologist. The median age of presentation was 5.2 years for this sub-group which was comprised of equal numbers of males and females. Ethnicity of this group included 6 African Americans, 1 Hispanic, 1 Caucasian, and 2 of unknown ancestry.

\section{Clinical Findings}

Table 2 lists the medical conditions that were present in the BEN cohort. No first degree relatives were noted to have a history of neutropenia. Five of the BEN patients had a history of hospitalization: 2 for febrile neutropenia (blood cultures remained negative), 1 each for mastoiditis, weight loss and neutropenia, and pneumonia. None of the hospitalized patients required intensive care unit (ICU) management. Three children with BEN also had eczema, 2 had attention deficit and hyperactivity disorder (ADHD), 2 had congenital heart disease, 1 
had developmental delay, and 1 had trisomy 21. Although one of the two patients with ADHD was on medications which might cause neutropenia, she had three CBCs prior to initiation of these medications which revealed an ANC between 300 and 500. All of the patients with congenital heart disease had structural defects, but no cardiomyopathy which might suggest Barth Syndrome. Two children with severe neutropenia were prescribed filgrastim for outpatient therapy for persistent neutropenia. One of those children had a robust response to filgrastim in which his white blood cell (WBC) count peaked at $15.2 \times 10^{9}$ cells/L, with $53 \%$ neutrophils and $15 \%$ bands. The response of the second patient who received filgrastim was not recorded.

\section{Laboratory Studies}

Table 3 shows an aggregate of the complete blood counts and immunoglobulins for the BEN cohort. The median ANC was $893 \times 10^{6}$ cells/L. Seventeen $(46 \%)$ had at least one ANC less than $500 \times 10^{6}$ cells/L. None of the individuals seen for less than 6 months or with a single follow-up visit had an ANC less than $500 \times 10^{6}$ cells/L. Of those with severe neutropenia, 8 were self-identified as African or African-American in heritage, 3 Hispanics, 2 Asians, 1 Caucasian, and 3 not reported. When analyzed by age groups, children less than 6 years-old had higher median WBC and lower ANC of $6.1 \times 10^{9}$ cells/L and $768 \times 10^{6}$ cells $/ \mathrm{L}$, respectively; 6-12 years-old, $3.2 \times 10^{9}$ and $833 \times 10^{6}$; and 13-18 years-old, $4.1 \times 10^{9}$ and $874 \times 10^{6}$ ( $\mathrm{p}<0.01$ for WBC, $\mathrm{p}=0.20$ for ANC). When analyzed by ethnicity, children of African descent versus other ethnicities had similar median WBC of 4.8 vs $6.2 \times 10^{9}$ cells/L, respectively ( $\mathrm{p}=0.19$ ), and similar median ANC of 813 vs $764 \times 10^{6}$ cells/L, respectively $(\mathrm{p}=0.18)$. Nine boys and 9 girls had hemoglobin levels between 11-12 g/dL. Among these 18 children with mild anemia, one had glucose-6-phosphate dehydrogenase deficiency, and the remainder had MCV between 70-82, suggesting iron deficiency and/or alpha-thalassemia trait, though alpha globin gene sequencing was not performed. Immunoglobulin levels were tested at least once in 17 (45.9\%) patients and all were within normal range. Five patients had bone marrow biopsies, 3 of which were normal and the others had focal $/ \mathrm{mild}$ hypocellularity with normal neutrophil maturation. Eight patients had negative antineutrophil antibody testing whereas 10 were negative for anti-nuclear antibodies. Two children had negative neutrophil elastase (ELANE) mutation testing; one child had a negative $H A X 1$ testing result.

\section{Discussion}

Through this analysis of the clinical data in children with BEN, we identified several demographic and laboratory characteristics of BEN in children that differed from BEN observed in previous adult studies. Nearly half of our BEN cohort had at least one ANC less than $500 \times 10^{6} \mathrm{cells} / \mathrm{L}$, and $86 \%$ had ANC between 500 and $1,000 \times 10^{6}$ cells/L. In contrast, the vast majority of adults with BEN had ANC $>1,000 \times 10^{6}$ cells/L. ${ }^{1,2}$ This low ANC was likely skewed by a selection bias in which pediatricians were more likely to refer a patient to the pediatric hematologist if they had a lower ANC. However, we believe that the lower ANC in our childhood cohort is an expected finding given that ANC rises with increasing age and the median age of our cohort was quite young at only 1.8 years upon initial presentation. The WBC trends in our BEN cohort are consistent with data in other healthy 
children, where younger children have higher total WBC counts with a higher proportion of lymphocytes, and as the children become older, the total WBC counts with lymphocyte percent decrease, while ANC increases. ${ }^{13}$ Second, our previous report in adult AfricanAmericans had highlighted a male predilection although this gender predominance was not seen in our study nor in a recent large study of Emirati Arabs. ${ }^{1,2}$ Lastly, there was no history of neutropenia in families of these children. This observation from genetically and ethnically diverse community of our referral area suggests that BEN may be inherited in a recessive manner or arise from another mechanism. Whether BEN in our cohort is similar to the BEN in other reports showing an autosomal dominance inheritance pattern is unknown, even though both appeared to be clinically benign.

Another well described feature of BEN in adults is that it almost exclusively occurs in individuals of African or Middle Eastern descent. ${ }^{1-5}$ However, only $60 \%$ of BEN individuals in our cohort were of African descent, and many were from ethnic backgrounds not previously described to have BEN. A possible explanation for the finding of non-traditional ethnicities being represented in our cohort is that in our diverse urban setting, patients who self-reported a non-traditional BEN ethnicity might have an ancestor with an African or Arab background. Seventy percent of the individuals in our cohort were less than 6 years old at presentation and as noted above, young children tend to have lower ANCs than adolescents and adults. Thus, another explanation is that this lower norm ANC for age might have included children of other ethnicities in our cohort of predominantly young children.

A challenge in chronicling the natural course of BEN is that since it is generally understood to be a benign variant, $\mathrm{BEN}$ is often not referred to a pediatric hematology clinic, or if it is referred may only warrant a single or brief monitoring. As a result, in our cohort there were 10 patients with less than 6 months of follow-up or a single visit. All of these patients were deemed by the pediatric hematologist of record, as well as an independent senior pediatric hematologist, to have findings consistent with BEN. As BEN implies chronic neutropenia, it was important to distinguish these patients from the excluded individuals whose neutropenia self-resolved or was transient, often after a viral infection. The ethnic and gender characteristics of this sub-cohort mirror that of the larger cohort and none of these patients had an episode of severe neutropenia (ANC less than $500 \times 10^{6}$ cells/L). As a result we believe that our cohort appropriately includes and excludes individuals with and without BEN, respectively, even those with limited follow-up. Recent reports have documented the association between DARC null state and leukopenia/neutropenia in those of African descent. Due to the retrospective nature of this study, red cell phenotyping for DARC or genetic studies for the known correlative SNPs were not performed, but certainly would further enhance our understanding of BEN. These genotype-phenotype correlation studies, however, still do not elucidate why polymorphisms at a red cell promoter cause leukopenia or neutropenia.

Finally, the children in our BEN cohort had an unremarkable clinical course. Their rates of infections are similar to other children, their rates of hospitalizations also appear to be low, and their associated medical conditions reflect those of the general pediatric population. ${ }^{14,15}$ Our study was not designed to calculate the prevalence of BEN in our outpatient hematology clinic. 
In summary, the natural history of BEN in children remained clinically benign. The rates of infection, hospitalization, and co-existing medical conditions appeared to be similar to healthy children. No medication was associated with BEN. Laboratory testing in BEN showed that other than mild anemia from possible iron deficiency or alpha-thalassemia trait, the remainder of the WBC indices and platelet counts were normal. This cohort of children with BEN highlights the ethnic heterogeneity, particularly in diverse communities where one should consider backgrounds besides African. In order to help pediatricians and pediatric hematologists tasked with evaluating a child with a potential diagnosis of BEN, we suggest work-up based on the severity and duration of neutrophil counts (refer to supplemental table with our recommended clinical practice guidelines).

\section{Supplementary Material}

Refer to Web version on PubMed Central for supplementary material.

\section{Acknowledgements}

The authors would like to thank Dr. Griffin Rodgers for the assistance with this manuscript and Xiongce Zhao for data analyses.

\section{References}

1. Hsieh MM, Everhart JE, Byrd-Holt DD, et al. Prevalence of neutropenia in the U.S. population: age, sex, smoking status, and ethnic differences. Ann Intern Med. Apr 3; 2007 146(7):486-92. [PubMed: 17404350]

2. Denic S, Showqi S, Klein C, et al. Prevalence, phenotype and inheritance of benign neutropenia in Arabs. BMC Blood Disorders. 2009; 9:3. doi:10.1186/1471-2326-9-3. [PubMed: 19323844]

3. Paz Z, Nails M, Ziv E. The genetics of benign neutropenia. Isr Med Assoc J. Oct; 2011 13(10):6259. [PubMed: 22097233]

4. Mason BA, Lessin L, Schechter GP. Marrow granulocyte reserves in black Americans. Hydrocortisone-induced granulocytosis in the "benign" neutropenia of the black. Am J Med. Aug; 1979 67(2):201-5. [PubMed: 463924]

5. Mant M, Gordon P, Akabutu J. Bone marrow granulocyte reserve in chronic benign idiopathic neutropenia. Clin Lab Haematol. 1987; 9(3):281-8. [PubMed: 3652640]

6. Mintz U, Sachs L. Normal granulocyte colony-forming cells in the bone marrow of Yemenite Jews with genetic neutropenia. Blood. Jun; 1973 41(6):745-51. [PubMed: 4712204]

7. Reich D, Nalls MA, Kao WH, et al. Reduced neutrophil count in people of African descent is due to a regulatory variant in the Duffy antigen receptor for chemokines gene. PLoS Genet. Jan.2009 5(1):e1000360. [PubMed: 19180233]

8. Thobakgale CF, Ndung'u T. Neutrophil counts in persons of African origin. Curr Opin Hematol. Jan; 2014 21(1):50-7. [PubMed: 24257098]

9. Rappoport N, Simon AJ, Lev A, et al. Correlation between 'ACKR1/DARC null' polymorphism and benign neutropenia in Yemenite Jews. Br J Haematol. Mar 26.2015 Epub ahead of print.

10. Shoenfeld Y, Ben-Tal O, Berliner S, et al. The outcome of bacterial infection in subjects with benign familial leukopenia (BFL). Biomed Pharmacother. 1985; 39(1):23-6. [PubMed: 4027348]

11. Haddy TB, Rana SR, Castro O. Benign ethnic neutropenia: what is a normal absolute neutrophil count? J Lab Clin Med. Jan; 1999 133(1):15-22. [PubMed: 10385477]

12. Lindqvist H, Carlsson G, Moell J, et al. Neutropenia in childhood: a 5-year experience at a tertiary center. Eur J Pediatr. Jun; 2015 174(6):801-7. [PubMed: 25435257]

13. Custer, J.; Rau, R., editors. The Harriet Lane Handbook. Eighteenth Edition. Mosby, Elsevier; Philadelphia, PA: 2009. 
14. National Center for Health Statistics. Health, United States, 2013: With Special Feature on Prescription Drugs. Hyattsville, MD.: 2014.

15. Adams, PF.; Hendershot, GE.; Marano, MA. Vital Health Stat. Vol. 10. National Center for Health Statistics; 1999. Current estimates from the National Health Interview Survey, 1996.. 


\section{Table 1}

\section{Demographic characteristics of BEN cohort}

\begin{tabular}{|c|c|c|c|}
\hline \multicolumn{2}{|c|}{ Characteristic } & \multirow{2}{*}{$\frac{\mathbf{N}}{19}$} & \multirow{2}{*}{$\frac{\%}{51.3}$} \\
\hline Gender & Male & & \\
\hline & Female & 18 & 48.6 \\
\hline \multirow{3}{*}{$\mathrm{Age}^{*}$} & Under 6 years & 26 & 70.2 \\
\hline & $6-12$ years & 5 & 13.5 \\
\hline & Over 12 years & 6 & 16.2 \\
\hline \multirow[t]{5}{*}{ Background } & African American/African & 22 & 59.5 \\
\hline & Caucasian & 4 & 10.8 \\
\hline & Hispanic & 3 & 8.1 \\
\hline & Asian & 2 & 5.4 \\
\hline & Not Documented & 6 & 16.2 \\
\hline
\end{tabular}

* Age at initial presentation to pediatric hematology clinic 


\section{Table 2}

Common reported symptoms from BN individuals

\begin{tabular}{|l|l|l|}
\hline Finding & Patients & \% Reporting \\
\hline Upper respiratory infection & 8 & 21.6 \\
\hline Rash & 6 & 16.2 \\
\hline Poor weight gain or weight loss & 4 & 10.8 \\
\hline Headache & 3 & 8.1 \\
\hline Oral ulcers & 3 & 8.1 \\
\hline Bone or joint pain & 2 & 5.4 \\
\hline Cervical lymphadenopathy & 2 & 5.4 \\
\hline Gingivitis & 1 & 2.7 \\
\hline Dental problems & 1 & 2.7 \\
\hline Night sweats or chills & 1 & 2.7 \\
\hline Dyspnea & 0 & 0 \\
\hline Delayed wound healing & 0 & 0 \\
\hline
\end{tabular}


Table 3

Complete blood counts and immunoglobulins from all individuals in the $\mathrm{BN}$ cohort

\begin{tabular}{|l|l|l|l|l|}
\hline Result & Units & $\mathbf{2 5}^{\text {th }}$ Quartile & Median & $\mathbf{7 5}^{\text {th }}$ Quartile \\
\hline White blood cell & $\times 10^{9}$ cells/L & 3.4 & 4.7 & 7.0 \\
\hline ANC & $\times 10^{6}$ cells/L & 344 & 627 & 1059 \\
\hline ALC & $\times 10^{6}$ cells/L & 1880 & 3070 & 4602 \\
\hline AMC & $\times 10^{6}$ cells/L & 331 & 449 & 645 \\
\hline Neutrophils & $\%$ & 6.7 & 13 & 24 \\
\hline Lymphocytes & $\%$ & 53.5 & 70.1 & 80.6 \\
\hline Monocytes & $\%$ & 7 & 9 & 11.4 \\
\hline Eosinophils & $\%$ & 1 & 4 & 5.6 \\
\hline Hemoglobin & $\mathrm{gm} / \mathrm{dL}$ & 11 & 11.7 & 12.7 \\
\hline Hematocrit & $\%$ & 32.1 & 34 & 36.8 \\
\hline Platelets & $\times 10^{9}$ cells/L & 192 & 266 & 329 \\
\hline Immunoglobulin G & $\mathrm{mg} / \mathrm{dL}$ & 499 & 649 & 1621 \\
\hline Immunoglobulin A & $\mathrm{mg} / \mathrm{dL}$ & 30.8 & 71.5 & 116.8 \\
\hline Immunoglobulin M & $\mathrm{mg} / \mathrm{dL}$ & 55 & 67.5 & 126 \\
\hline
\end{tabular}

* Based on 175 CBCs and 17 Immunoglobulin levels for 37 patients 